\title{
Disruption of calcium transfer from ER to mitochondria links alterations of mitochondria-associated ER membrane integrity to hepatic insulin resistance
}

\author{
Jennifer Rieusset $^{1} \cdot$ Jeremy Fauconnier $^{2} \cdot$ Melanie Paillard $^{1} \cdot$ Elise Belaidi $^{1}$. \\ Emily Tubbs $^{1}$ - Marie-Agnès Chauvin ${ }^{1}$ - Annie Durand ${ }^{1}$ • Amélie Bravard ${ }^{1}$. \\ Geoffrey Teixeira ${ }^{1} \cdot$ Birke Bartosch $^{3} \cdot$ Maud Michelet $^{3} \cdot$ Pierre Theurey $^{1}$. \\ Guillaume Vial $^{1}$ - Marie Demion ${ }^{2}$ Emilie Blond ${ }^{1,4} \cdot$ Fabien Zoulim $^{3,4}$. \\ Ludovic Gomez $^{1}$ - Hubert Vidal ${ }^{1}$ • Alain Lacampagne ${ }^{2}$ - Michel Ovize ${ }^{1,4}$
}

Received: 25 June 2015 / Accepted: 17 November 2015 /Published online: 10 December 2015

(C) Springer-Verlag Berlin Heidelberg 2015

\begin{abstract}
Aims/hypothesis Mitochondria-associated endoplasmic reticulum membranes (MAMs) are regions of the endoplasmic reticulum (ER) tethered to mitochondria and controlling calcium $\left(\mathrm{Ca}^{2+}\right)$ transfer between both organelles through the complex formed between the voltage-dependent anion channel, glucose-regulated protein 75 and inositol 1,4,5-triphosphate receptor (IP3R). We recently identified cyclophilin D (CYPD) as a new partner of this complex and demonstrated a new role for MAMs in the control of insulin's action in the
\end{abstract}

Jeremy Fauconnier and Melanie Paillard contributed equally to this work.

An earlier version of this paper was published in Hepatology in 2013 but was retracted after errors in the presentation of western blot data became apparent (DOI: 10.1002/hep.26189). At the authors' request their institutions (Research Offices of Inserm, Lyon and Montpellier Universities) checked the integrity of all the raw data, concluding that the mistakes that led to the retraction were a result of honest error. The images presented here have all passed image forensics screening.

Electronic supplementary material The online version of this article (doi:10.1007/s00125-015-3829-8) contains peer-reviewed but unedited supplementary material, which is available to authorised users.

Jennifer Rieusset

jennifer.rieusset@univ-lyon1.fr

1 Inserm UMR-1060, Laboratoire CarMeN, Université Lyon 1, 165 chemin du grand Revoyet, BP12, 69921 Oullins cedex, France

2 Inserm U1046-CNRS UMR-9214, PhyMedExp, Université Montpellier, Montpellier, France

3 Inserm UMR-1052, Centre de recherche en Cancérologie de Lyon, Université Lyon 1, Lyon, France

4 Hospices Civils de Lyon, Lyon, France liver. Here, we report on the mechanisms by which disruption of MAM integrity induces hepatic insulin resistance in $C y p D$ (also known as Ppif)-knockout (KO) mice.

Methods We used either in vitro pharmacological and genetic inhibition of CYPD in HuH7 cells or in vivo loss of CYPD in mice to investigate ER-mitochondria interactions, inter-organelle $\mathrm{Ca}^{2+}$ exchange, organelle homeostasis and insulin action.

Results Pharmacological and genetic inhibition of CYPD concomitantly reduced ER-mitochondria interactions, inhibited inter-organelle $\mathrm{Ca}^{2+}$ exchange, induced ER stress and altered insulin signalling in $\mathrm{HuH} 7$ cells. In addition, histaminestimulated $\mathrm{Ca}^{2+}$ transfer from ER to mitochondria was blunted in isolated hepatocytes of $C y p D-\mathrm{KO}$ mice and this was associated with an increase in ER calcium store. Interestingly, disruption of inter-organelle $\mathrm{Ca}^{2+}$ transfer was associated with ER stress, mitochondrial dysfunction, lipid accumulation, activation of c-Jun N-terminal kinase (JNK) and protein kinase C (PKC) $\varepsilon$ and insulin resistance in liver of $C y p D-K O$ mice. Finally, CYPD-related alterations of insulin signalling were mediated by activation of $\mathrm{PKC} \varepsilon$ rather than JNK in $\mathrm{HuH} 7$ cells. Conclusions/interpretation Disruption of IP3R-mediated $\mathrm{Ca}^{2+}$ signalling in the liver of CypD-KO mice leads to hepatic insulin resistance through disruption of organelle interaction and function, increase in lipid accumulation and activation of PKCE. Modulation of ER-mitochondria $\mathrm{Ca}^{2+}$ exchange may thus provide an exciting new avenue for treating hepatic insulin resistance.

Keywords Calcium signalling · Cyclophilin D - Endoplasmic reticulum $\cdot$ Inositol 1,4,5-triphosphate receptor $\cdot$ Insulin resistance $\cdot$ Liver $\cdot$ Mitochondria $\cdot$ Mitochondria-associated endoplasmic reticulum membranes $\cdot \mathrm{PKC} \varepsilon$ 


$\begin{array}{ll}\text { Abbreviations } \\ \text { CsA } & \text { Ciclosporin (cyclosporin A) } \\ \text { CYPD } & \text { Cyclophilin D } \\ \text { DAG } & \text { Diacylglycerol } \\ \text { EIF2 } \alpha & \text { Eukaryotic translation initiation factor } 2 \alpha \\ \text { ER } & \text { Endoplasmic reticulum } \\ \text { GRP75 } & \text { Glucose-regulated protein } 75 \\ \text { IP3R } & \text { Inositol 1,4,5-triphosphate receptor } \\ \text { JNK } & \text { c-Jun N-terminal kinase } \\ \text { KO } & \text { Knock-out } \\ \text { MAM } & \text { Mitochondrial-associated endoplasmic reticulum } \\ & \text { membrane } \\ \text { MFN2 } & \text { Mitofusin 2 } \\ \text { PKB } & \text { Protein kinase B } \\ \text { PKC } \varepsilon & \text { Protein kinase C epsilon } \\ \text { PLA } & \text { Proximity ligation assay } \\ \text { PTP } & \text { Permeability transition pore } \\ \text { SERCA2 } & \text { Sarco(endo)plasmic reticulum } \mathrm{Ca}^{2+} \text { ATPase 2 } \\ \text { SiRNA } & \text { Small interfering RNA } \\ \text { TG } & \text { Triacylglycerol } \\ \text { VDAC } & \text { Voltage-dependent anion channel } \\ \text { WT } & \text { Wild-type }\end{array}$

\section{Introduction}

Mitochondria and endoplasmic reticulum (ER) are organised as a network with specific contact points, referred to as mitochondrial-associated ER membranes (MAMs), which play a pivotal role in calcium $\left(\mathrm{Ca}^{2+}\right)$ signalling and energy metabolism [1]. Efficient $\mathrm{Ca}^{2+}$ transmission from the ER to mitochondria is mediated through the interaction of the voltage-dependent anion channel (VDAC) of the outer mitochondrial membrane with the inositol-1,4,5-triphosphate receptor (IP3R) on the ER via the chaperone glucose-regulated protein 75 (GRP75) [2]. Mitochondrial $\mathrm{Ca}^{2+}$ uptake is essential for the regulation of both mitochondrial metabolism and ER homeostasis [3], and alteration of ER-mitochondria crosstalk may result in a disruption of inter-organelle $\mathrm{Ca}^{2+}$ transfer [4] and subsequent ER stress [5]. Under certain conditions excessive $\mathrm{Ca}^{2+}$ entry into the mitochondrial matrix may be detrimental, causing the opening of the permeability transition pore (PTP) and cell death [6]. Therefore, tight control of $\mathrm{Ca}^{2+}$ exchange between ER and mitochondria is required to regulate vital functions and metabolic homeostasis.

The molecular and functional characterisation of MAMs in physiological and pathological conditions has improved in the last few years, highlighting unexpected roles for MAMs in cellular signalling [7]. Particularly, we recently demonstrated that cyclophilin D (CYPD), a mitochondrial protein known to modulate the opening of the PTP [8], also interacts with the VDAC-GRP75-IP3R complex at the MAM interface in both heart [9] and liver [10]. In cardiomyocytes, we found that the loss of CYPD reduced mitochondrial $\mathrm{Ca}^{2+}$ overload by depressing ER-mitochondria interactions and protected cells against lethal reperfusion injury [9], suggesting that CYPD regulates $\mathrm{Ca}^{2+}$ transfer from ER to mitochondria. In liver, the loss of CYPD reduced organelle interactions and induced hepatic insulin resistance, pointing to a new role of MAM integrity in the control of insulin's action [10]. Whereas other studies in mice also suggest a role for MAMs in the control of glucose homeostasis [11-14], the mechanisms by which disruption of MAMs alters insulin signalling are unknown.

Based on the role of MAMs in $\mathrm{Ca}^{2+}$ transfer from ER to mitochondria, we hypothesised that a disruption of $\mathrm{Ca}^{2+}$ transfer between both organelles could contribute to hepatic insulin resistance. To this aim, using pharmacological and genetic loss of function approaches both in vivo and in vitro, we investigated whether the disruption of $\mathrm{Ca}^{2+}$ transfer from ER to mitochondria could link MAM alterations to hepatic insulin resistance in $C y p D$ (also known as Ppif)-knockout (KO) mice.

\section{Methods}

Cell culture $\mathrm{HuH} 7$ cells were a gift from G. Mithieux's laboratory (Inserm U855, Lyon, France) and were free of mycoplasma. They were cultured as previously described [10] and stimulated either with CYPD (ciclosporin [cyclosporin A, CsA] or NIM811) or with IP3R (2-APB and Xestospongin C) pharmacological inhibitors. Inhibition of CYPD was also achieved by silencing of CYPD. See electronic supplementary material (ESM) Methods for further details. For measurement of insulin signalling, cells were depleted in serum for $3 \mathrm{~h}$ before incubation with insulin $\left(10^{-7} \mathrm{~mol} / \mathrm{l}, 15 \mathrm{~min}\right)$.

Animals CYPD-KO mice on a C57Bl/6/SV129 genetic background were a gift from S. J. Korsmeyer's laboratory (Boston, MA, USA) [15]. Both male wild-type (WT) and CYPD-KO mice were obtained by homozygous intercross in our laboratory. All experiments were performed on mice of 18-22 weeks of age, and were conducted in accordance with institutional guidelines for the care and use of laboratory animals, and a regional ethics committee has approved all procedures. No randomisation or blinding were performed. No data samples or animals were excluded from the study.

$\mathrm{Ca}^{2+}$ measurements Using confocal fluorescence imaging, we evaluated variations in the time course of mitochondrial $\mathrm{Ca}^{2+}$ content simultaneously with intra-ER $\mathrm{Ca}^{2+}$ in a native cell environment in the presence of extracellular calcium $\left(1.8 \mathrm{mmol} / 1 \mathrm{CaCl}_{2}\right)$ [16]. To measure mitochondrial $\mathrm{Ca}^{2+}$, cells were loaded with Rhod-2 AM $(3 \mu \mathrm{mol} / 1)$ whereas the measurement of $\mathrm{ER} \mathrm{Ca}^{2+}$ was performed using a low-affinity $\mathrm{Ca}^{2+}$ indicator, Fluo-5N (5 $\mu \mathrm{mol} / \mathrm{l}$; ThermoFisher Scientific, MA, USA). For measurement of absolute cytosolic $\mathrm{Ca}^{2+}$ 
levels, cells were loaded with $1 \mu \mathrm{mol} / \mathrm{l}$ of Fura2-AM and pluronic acid (both from ThermoFisher Scientific). See ESM Methods for further details.

ER-mitochondria interactions ER-mitochondria interactions were measured both by subcellular fractionation and by in situ proximity ligation assay (PLA), as previously described and thoroughly validated [10]. See ESM Methods for further details.

Real-time PCR mRNA levels were measured by real-time RT-PCR. See ESM Methods for further details.

Western blotting Protein expression was analysed by SDSPAGE. See ESM Methods for further details.

Hyperinsulinaemic-euglycaemic clamp Insulin sensitivity of mice was measured during a hyperinsulinaemiceuglycaemic clamp. See ESM Methods for further details.

Primary hepatocytes Primary mouse hepatocytes were isolated via a modified collagenase perfusion method, as described previously [17]. See ESM Methods for further details.

Mitochondrial respiration Mitochondrial respiration was measured in intact or permeabilised primary hepatocytes. See ESM Methods for further details.

Hepatic lipid content Hepatic triacylglycerol (TG) and diacylglycerol (DAG) content were measured by spectrophotometry. See ESM Methods for further details.

PKC $\varepsilon$ activity $\mathrm{PKC} \varepsilon$ activity in liver was determined by western blot based on the membrane translocation. See ESM Methods for further details.

Statistical analysis Results are expressed as mean \pm SEM. Student's $t$-test was used to analyse the difference between control and experimental groups. Statistically significant differences were assessed with a one-way ANOVA with a Newman-Keuls post hoc test when three or more groups were compared. A $p$ value $<0.05$ was considered as statistically significant.

\section{Results}

Pharmacological and genetic inhibition of CYPD inhibits $\mathrm{Ca}^{2+}$ transfer from ER to mitochondria in $\mathrm{HuH7}$ cells We recently demonstrated by in situ PLA that CYPD interacted with the VDAC1-GRP75-IP3R1 calcium-channelling complex $[9,10]$. Here, we challenged the interactions of CYPD with this complex using pharmacological and genetic loss of function studies, and investigated the repercussions on both ER-mitochondria interactions and $\mathrm{Ca}^{2+}$ transfer. As CYPD inhibitors we used CsA and NIM811 (a CsA derivative devoid of immunosuppressive activity), which are both known to detach CYPD from the inner mitochondrial membrane [18]. Treatment of HuH7 cells with CsA or NIM811 significantly inhibited CYPD-IP3R1 interactions (Fig. 1a). Reduction of CYPD expression using specific small interfering RNA (siRNA) (Fig. 1b) also significantly decreased the interactions between CYPD and IP3R1 (Fig. 1c).

Using organelle-targeted fluorescent dye, we then measured $\mathrm{Ca}^{2+}$ flux in $\mathrm{HuH} 7$ cells under histamine stimulation $(100 \mu \mathrm{mol} / \mathrm{l})$. Histamine binding to its receptor induces inositol-1,4,5-triphosphate elevation and activates IP3R, causing $\mathrm{Ca}^{2+}$ release from ER stores. Specificity and non-overlapping of the $\mathrm{Ca}^{2+}$-sensitive dyes is illustrated in ESM Fig. 1. Histamine application rapidly reduced $\mathrm{ER} \mathrm{Ca}^{2+}$ stores (Fig. 2a) and simultaneously increased mitochondrial $\mathrm{Ca}^{2+}$ content (Fig. 2b), illustrating transfer of $\mathrm{Ca}^{2+}$ from the ER to the mitochondria. Importantly, after NIM811 treatment or CYPD silencing, histamine was unable to induce $\mathrm{Ca}^{2+}$ transfer from ER to mitochondria, as illustrated by the absence of mitochondrial $\mathrm{Ca}^{2+}$ accumulation in both situations (Fig. 2a, b).

Inhibition of CYPD function induces ER stress and alters insulin response in $\mathrm{HuH7}$ cells Next, we measured the consequences of CYPD inhibition on both ER and mitochondrial homeostasis and on insulin signalling in $\mathrm{HuH} 7$ cells. NIM811 treatment significantly increased mRNA levels of GRP78, $X B P 1 S$ and CHOP (also known as DDIT3) (Fig. 3a), as well as the protein level of GRP78 and the phosphorylation of eukaryotic translation initiation factor $2 \alpha$ (EIF2 $\alpha$ ) (Fig. 3b), indicative of ER stress. However, this treatment had no effect on the mRNA levels of HSP10 and HSP60 (Fig. 3a), two markers of mitochondrial stress that are increased during the mitochondrial unfolded protein response [19]. Inhibition of CYPD using CsA reproduced the same effects (ESM Fig. 2a, b). Finally, the partial invalidation of CYPD expression by specific siRNA also induced ER stress markers (Fig. 3c).

NIM811 treatment also significantly reduced insulinstimulated protein kinase B (PKB) phosphorylation (Fig. 3d) and induced PEPCK mRNA expression (ESM Fig. 2c) in $\mathrm{HuH} 7$ cells. Inhibition of CYPD using CsA reproduced all these effects (ESM Fig. 2d, e). In addition, the partial invalidation of CYPD expression by siRNA also altered insulin signalling (Fig. 3e) and induced PEPCK expression (ESM Fig. 2f). To strengthen the link between IP3R-mediated $\mathrm{Ca}^{2+}$ signalling, ER homeostasis and insulin signalling, we investigated whether pharmacological inhibition of IP3R could alter insulin signalling independently of CYPD. Both 2-APB and Xestospongin $\mathrm{C}$ treatments, which antagonise the calciumreleasing action of inositol-1,4,5-trisphosphate at the receptor 
a Negative control
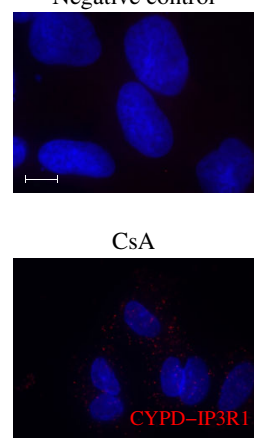

b

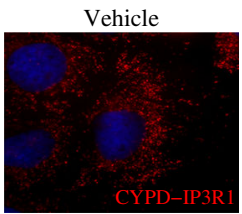

NIM811

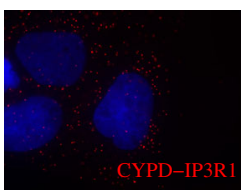

C

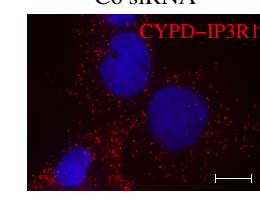

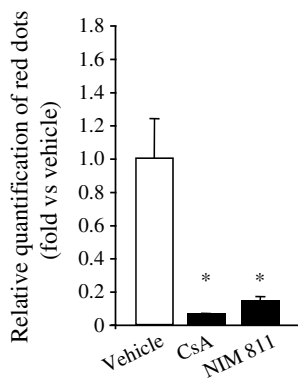

CYPD SIRNA

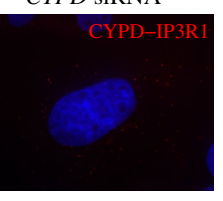

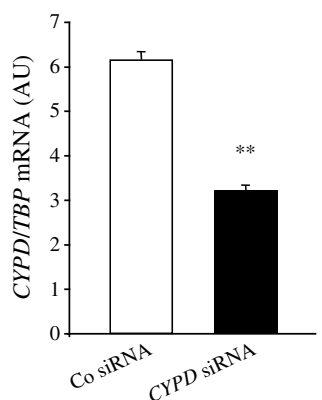

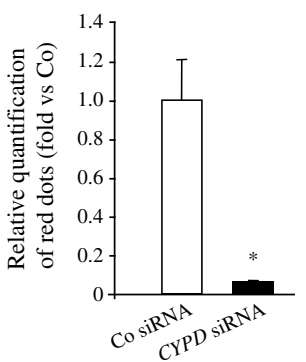

Fig. 1 Pharmacological and genetic inhibition of CYPD alters its interaction with the VDAC1-GRP75-IP3R1 complex. (a, c) Representative images (scale bar, $20 \mu \mathrm{m}$ ) and quantitative analysis of CYPD-IP3R1 interactions measured by in situ PLA in $\mathrm{HuH} 7$ cells, following treatment with either CsA or NIM811 (2 $\mu \mathrm{mol} / 1,16 \mathrm{~h})(\mathbf{a})$, or silencing of $C Y P D$ $(25 \mathrm{nmol} / \mathrm{l}, 48 \mathrm{~h})(\mathbf{c})$. Nuclei appear in blue and PLA-specific signals in red. Magnification $\times 63$. ${ }^{*} p<0.05$ vs control, $n=3$. Co, control. (b) Measurement of CYPD mRNA levels by real-time PCR in $\mathrm{HuH7}$ cells silenced for $C Y P D .{ }^{*} * p<0.001, n=3$

$\mathbf{a}$

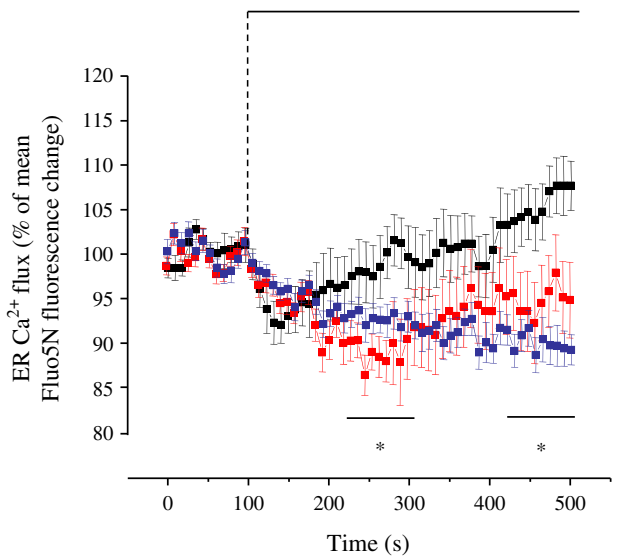

Fig. 2 Pharmacological and genetic inhibition of CYPD alters IP3Rmediated $\mathrm{Ca}^{2+}$ transfer between ER and mitochondria in $\mathrm{HuH} 7$ cells. $(\mathbf{a}, \mathbf{b})$ Measurement by confocal imaging of $\mathrm{Ca}^{2+}$ flux into ER (a) and mitochondria (b) in histamine-stimulated $\mathrm{HuH} 7$ cells, in control situation (Co, black curve), after NIM811 treatment ( $2 \mu \mathrm{mol} / \mathrm{l}, 16 \mathrm{~h}$, red curve) or level, significantly induced ER stress (Fig. 4a, b) and reduced insulin-stimulated PKB phosphorylation (Fig. 4c, d) in $\mathrm{HuH7}$ cells.

Loss of CYPD in mice alters $\mathrm{Ca}^{2+}$ transfer from ER to mitochondria in isolated hepatocytes We previously found that ER-mitochondria interactions were reduced in liver of CypD-KO mice [10]. We confirmed in this study our initial observation using an independent group of mice (Fig. 5a) and further analysed the composition of MAM fractions of WT and CypD-KO mice. We found no significant modification of IP3R1, VDAC1, GRP75, mitofusin 2 (MFN2) or sarco(endo)plasmic reticulum $\mathrm{Ca}^{2+}$ ATPase 2 (SERCA2) protein level in MAM fractions of CypD-KO mice compared with WT mice (Fig. 5b). This suggests that loss of CYPD induces a dissociation of organelles rather than a change in MAM protein composition. We then examined inter-organelle $\mathrm{Ca}^{2+}$ flux in isolated hepatocytes of CypD-KO mice, following the same experimental protocol performed in HuH7 cells. In WT hepatocytes, histamine induced $\mathrm{Ca}^{2+}$ release from the ER, immediately followed by mitochondrial $\mathrm{Ca}^{2+}$ uptake (Fig. 5c, d). This histaminestimulated $\mathrm{Ca}^{2+}$ transfer from the ER to mitochondria was completely abolished in CypD-KO hepatocytes (Fig. 5c, d). This occurs in the absence of a modification of Serca2b (also known as Atp2a2) and Mcu mRNA levels in liver of CypD-KO mice (ESM Fig. 3a). Together, these results confirm in a more physiological model that CYPD participates in IP3-mediated ER-tomitochondria $\mathrm{Ca}^{2+}$ transfer in hepatocytes.

We also measured cytoplasmic $\mathrm{Ca}^{2+}$ in response to a discharge of ER store by thapsigargin, both in the presence and absence of extracellular $\mathrm{Ca}^{2+}$. In both conditions, thapsigargin-sensitive $\mathrm{Ca}^{2+}$ stores were significantly higher in CypD-KO mice compared with WT mice (Fig. 5e, f),

b

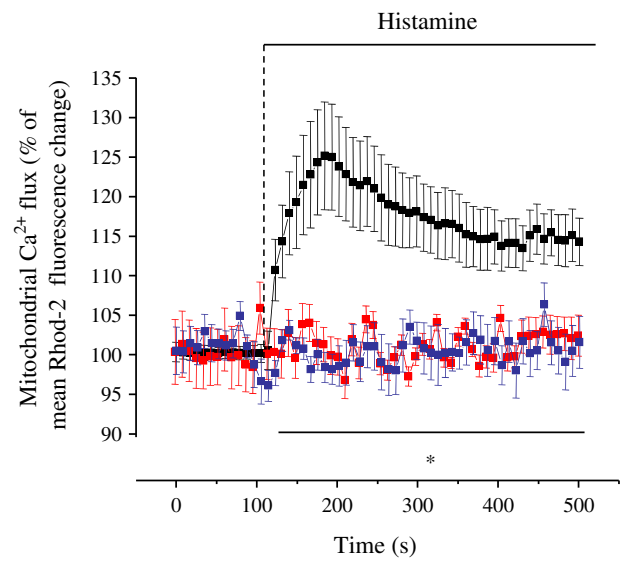

after siRNA-mediated CYPD silencing ( $25 \mathrm{nmol} / \mathrm{l}, 48 \mathrm{~h}$, blue curve). Curves represent the time course of $\mathrm{Ca}^{2+}$ exchange between ER and mitochondria for $500 \mathrm{~s}$ after histamine stimulation $(100 \mu \mathrm{mol} / \mathrm{l})$, in the presence of extracellular $\mathrm{Ca}^{2+}$. Mean $\mathrm{Ca}^{2+}$ flux was normalised to fluorescence values prior to histamine application. ${ }^{*} p<0.05$ vs control, $n=18$ 
a
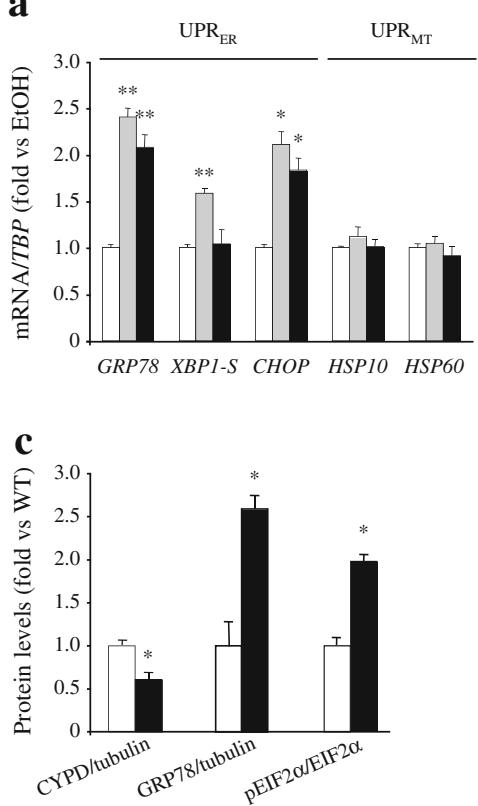

d
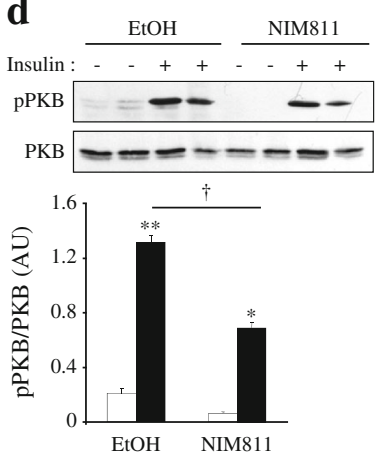
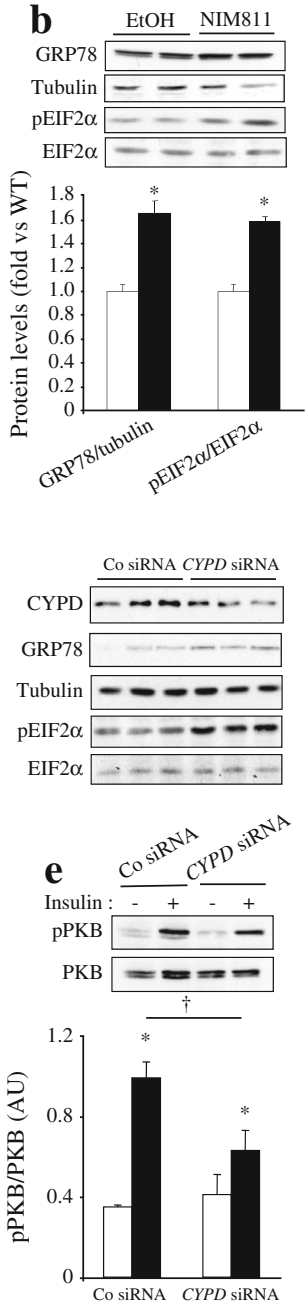

$\mathbf{a}$
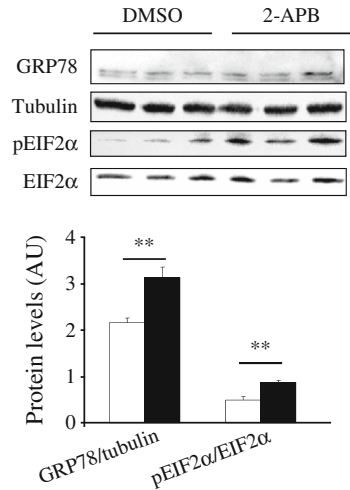

c
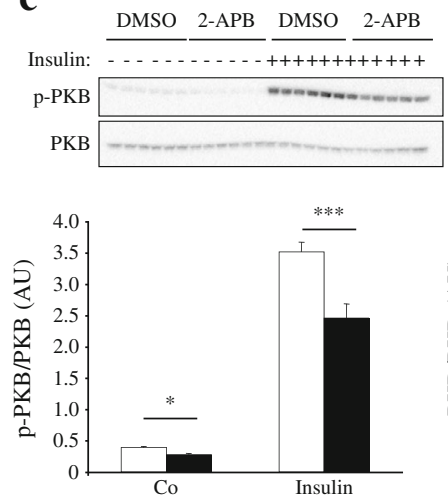

b
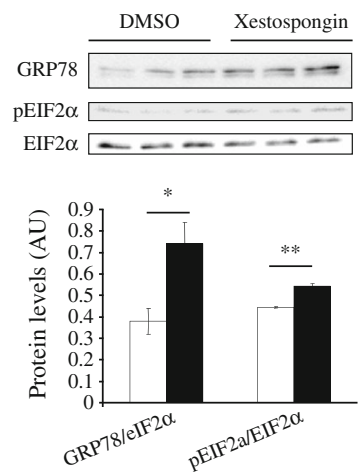

d
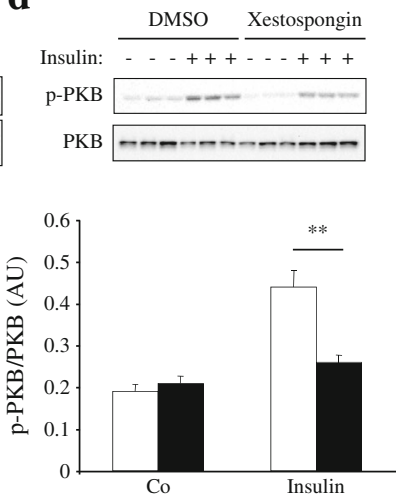

Fig. 4 Pharmacological inhibition of IP3R induces ER stress and alters insulin signalling in $\mathrm{HuH7}$ cells. (a-d) Representative western blots and quantitative analysis of both ER stress markers $(\mathbf{a}, \mathbf{b})$ and insulin-stimulated $\mathrm{PKB}$ phosphorylation (c, d) in $\mathrm{HuH} 7$ cells treated (black bars) or not (white bars) with 2-APB (a and c, $50 \mu \mathrm{mol} / 1,18 \mathrm{~h}$ ) or Xestospongin (b and d, $1 \mu \mathrm{mol} / 1,18 \mathrm{~h}$ ). Co, control. ${ }^{*} p<0.05,{ }^{* *} p<0.01$ and *** $p<0.005, n=3-6$

found increased mRNA levels of Grp78 and Chop in the liver of CypD-KO mice, without any change in Hsp10 and Hsp60 expression (Fig. 6a). In addition, the phosphorylation of PERK, EIF $2 \alpha$ and of JNK (a serine/threonine kinase involved in ER stress-induced hepatic insulin resistance [20]) was increased in liver of CypD-KO mice (Fig. 6b), confirming hepatic ER stress.

As $\mathrm{Ca}^{2+}$ import into mitochondria affects mitochondrial bioenergetics [21, 22], we studied mitochondrial respiration in either permeabilised (glutamate/malate) or intact $(20 \mathrm{mmol} /$ 1 glucose) primary hepatocytes of both WT and CypD-KO mice, in order to maintain ER mitochondria cross-talk. As shown in Fig. 6c, d, oxygen consumption was significantly reduced in CypD-KO hepatocytes, compared with WT hepatocytes.

Loss of CYPD in mice increases lipid accumulation and PKC activity and induces hepatic insulin resistance We previously reported that $C y p D-\mathrm{KO}$ mice are glucose intolerant, insulin resistant and showed increased gluconeogenesis, based on tolerance tests [10]. Here, we further performed hyperinsulinaemic-euglycaemic clamp to confirm hepatic 
a

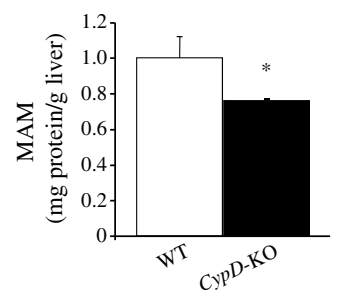

c

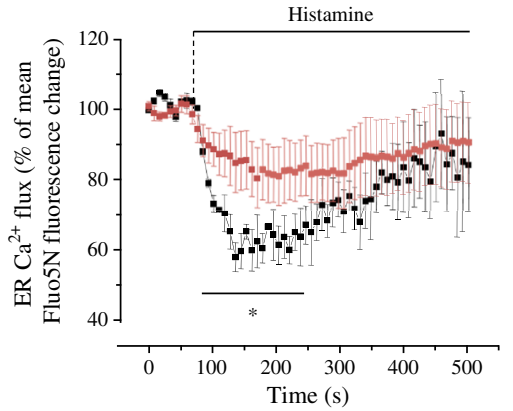

e

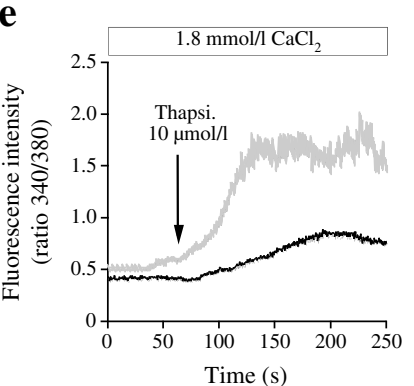

b $\quad{ }_{\text {WT }}^{\text {CypD }}$

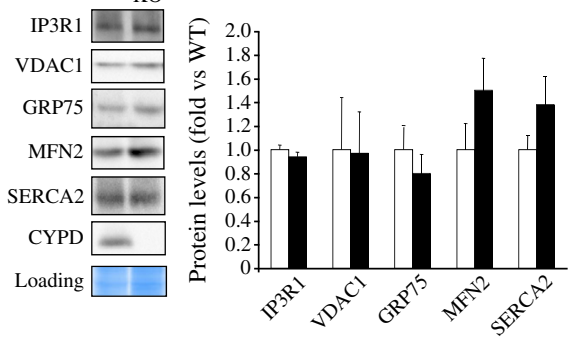

d

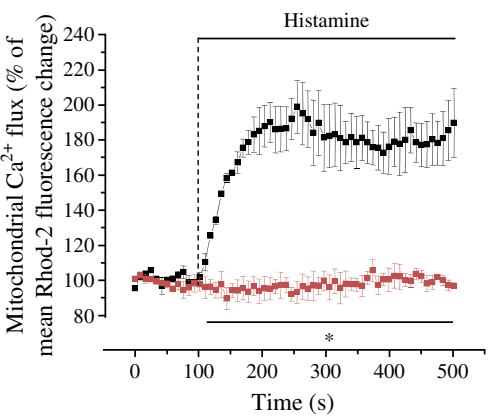

f

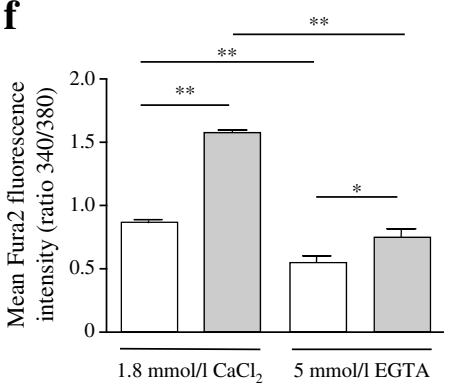

mice. Experiments and presentation of the results are as described in Fig. 2. ${ }^{*} p<0.05$ for $C y p D-K O$ vs WT $(n=12)$. (e, f) Representative curves (e) and quantitative analysis (f) of cytosolic $\mathrm{Ca}^{2+}$ (Fura2), following depletion of ER $\mathrm{Ca}^{2+}$ storage by thapsigargin (Thapsi.; $10 \mu \mathrm{mol} / \mathrm{l}$ ), in the presence $\left(1.8 \mathrm{mmol} / 1 \mathrm{CaCl}_{2}\right)$ or absence $(5 \mathrm{mmol} / 1 \mathrm{EGTA})$ of extracellular $\mathrm{Ca}^{2+}$ in WT (black curve and white bars) and CypD-KO (grey curve and grey bars) mice. ${ }^{*} p<0.01$ and ${ }^{*} p<0.001$ for indicated comparisons $(n=$ 16-25)

the lipid staining (Fig. 7d), as well as TG and total DAG levels (Fig. 7e), were significantly increased in the liver of $C y p D-K O$ mice. As PKC $\varepsilon$ was shown to be involved in DAG-mediated hepatic insulin resistance [23], we analysed its activity by measuring its translocation from cytosol to membrane. Consistent with DAG accumulation, $\mathrm{PKC} \varepsilon$ activity was induced in the livers of $C y p D-\mathrm{KO}$ mice (Fig. $7 \mathrm{f}$ ). Furthermore, the mRNA levels of genes related to $\beta$-oxidation $(C p t l a)$ were decreased in liver of $C y p D-\mathrm{KO}$ mice, whereas the expression of both lipogenic genes (Srebplc [also known as Srebf1], Srebpla, Fasn, Acaca) and genes involved in lipid export (Apob, Mttp) were increased (Fig. 7g). The expression of neither Mlycd nor Dgat 2 was modified in liver of $C y p D-K O$ mice compared with WT mice. Altogether, these results suggest that lipid accumulation in liver of $C y p D-\mathrm{KO}$ mice is likely related to both a 


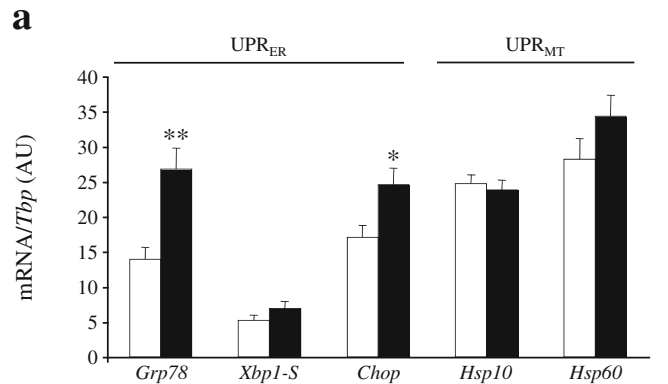

b

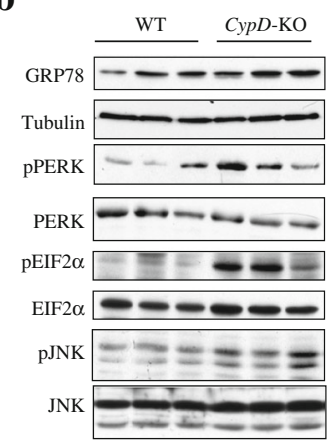

c
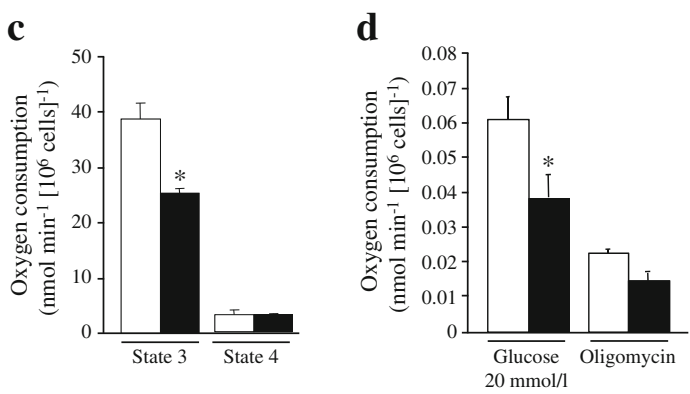

Fig. 6 Loss of CYPD induces hepatic ER stress and mitochondrial dysfunction in mice. (a, b) mRNA (a) and protein (b) levels of ER and mitochondria stress markers in liver of WT and CypD-KO mice. ${ }^{*} p<0.05$ and $* * p<0.01$ vs WT mice $(n=3-10)$. UPR, unfolded protein response; MT mitochondria; tub, tubulin. (c, d) Oxygen consumption measured in either permeabilised primary hepatocytes (c, in response to $5 \mathrm{mmol} / \mathrm{l}$ glutamate $+25 \mathrm{mmol} / \mathrm{l}$ malate stimulation in both states 3 [+ $1 \mathrm{mmol} / \mathrm{l} \mathrm{ADP}]$ and 4 [+ oligomycin]) or in intact hepatocytes (d, in the presence of $20 \mathrm{mmol} / \mathrm{l}$ glucose and after addition of oligomycin) from WT and $C y p D-K O$ mice. White bars, WT mice; black bars, $C y p D-\mathrm{KO}$ mice. ${ }^{*} p<0.05$ vs WT $(n=4)$

reduction in lipid oxidation and an increase in lipid storage, the latter being mainly associated with increased de novo lipogenesis.

\section{Role of PKC $\varepsilon$ in CYPD-related alteration of insulin} signalling Both JNK and $\mathrm{PKC} \varepsilon$ enzymes, which are increased in liver of $C y p D-\mathrm{KO}$ mice, are potential mediators of hepatic insulin resistance [20,23]. To discriminate between the two, we measured the effect of CYPD silencing on insulinstimulated $\mathrm{PKB}$ phosphorylation in the presence or absence of JNK and PKC $\varepsilon$ inhibitors. Inhibition of JNK with SP600125 did not modify $C Y P D$ siRNA-induced alteration of insulin-
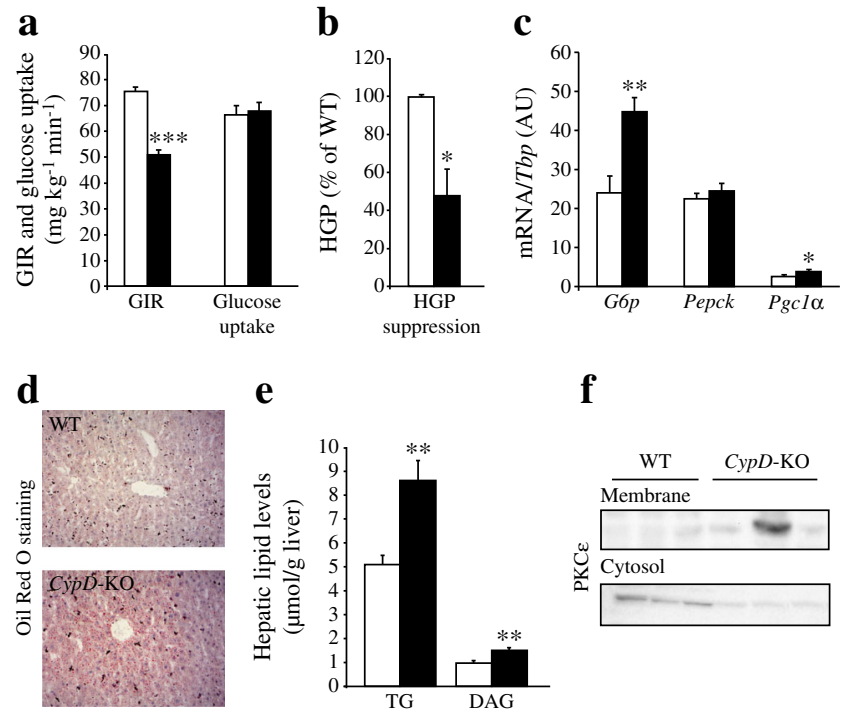

e

$\mathbf{f}$
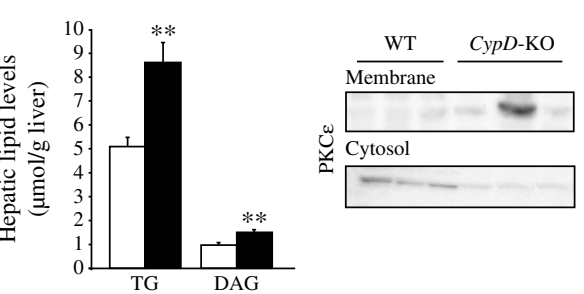

g

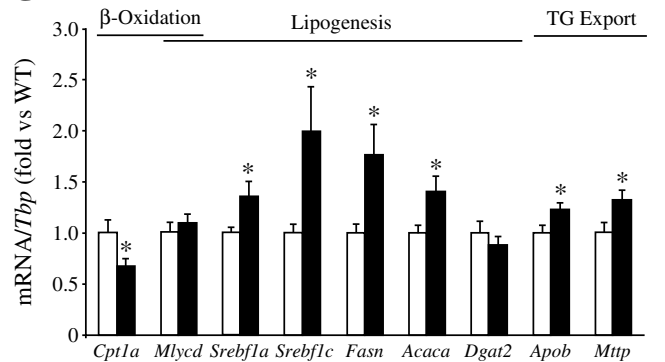

Fig. 7 Loss of CYPD induces hepatic insulin resistance and alterations of lipid homeostasis in mice. (a, b) Glucose infusion rate (GIR) and glucose uptake (a), as well as suppression of hepatic glucose production (HGP) (b) was measured under a hyperinsulinaemic-euglycaemic clamp in WT and CypD-KO mice $(n=4)$. (c) Expression of gluconeogenic genes G6p, Pepck and Pgcl $\alpha$ (also known as Ppargcla) in the liver of WT and CypD-KO mice $(n=10)$. (d) Liver sections were stained with Oil Red O. Original magnification, $\times 20$. (e) Hepatic TG and DAG content in liver of WT and CypD-KO mice $(n=6)$. (f) Measurement of PKC $\varepsilon$ activation in cytosolic and membrane fractions of liver of WT and $C y p D-K O$ mice $(n=$ 3). (g) mRNA levels of genes related to $\beta$-oxidation, lipogenesis and TG export in liver of WT and CypD-KO mice $(n=10)$. White bars, WT mice; black bars, $C y p D-\mathrm{KO}$ mice. ${ }^{*} p<0.05,{ }^{*} p<0.01$ and $* * * p<0.001$ vs WT

stimulated PKB phosphorylation (Fig. 8a), whereas inhibition of $\mathrm{PKC} \varepsilon$ activity, using a specific peptide inhibitor of $\mathrm{PKC} \varepsilon$, prevented the reduction of insulin-stimulated PKB phosphorylation induced by CYPD silencing in $\mathrm{HuH7}$ cells (Fig. 8b). Together, these data suggest a predominant role for $\mathrm{PKC} \varepsilon$, rather than JNK, in CYPD-related alterations of insulin signalling, at least in vitro.

\section{Discussion}

Hepatic insulin resistance is a principal component of type 2 diabetes, but the cellular and molecular mechanisms responsible for its pathogenesis are only partly known. We recently proposed a role for MAM integrity in the control of hepatic 
$\mathbf{a}$

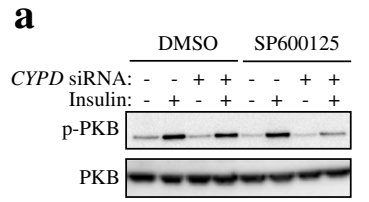

b
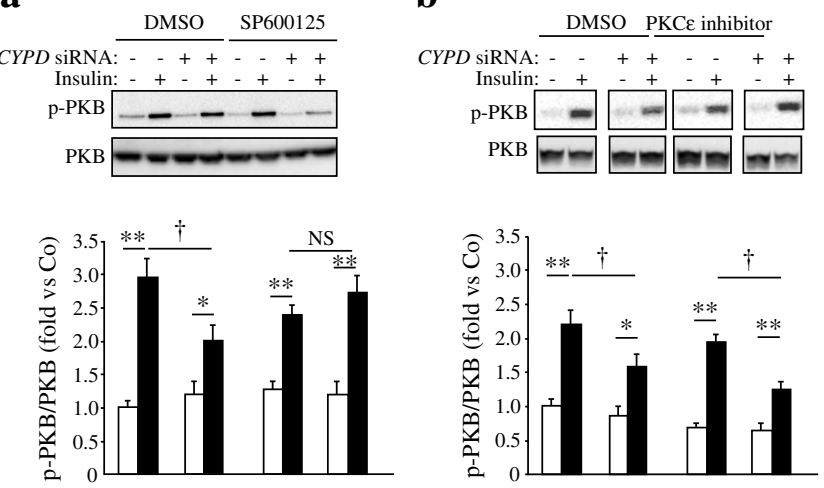

CYPD SiRNA: - - + + - + + CYPD siRNA: - $+++{ }_{+}++$ SP600125: - - - + + + + PKCE inhibitor: - - - + + + +

C

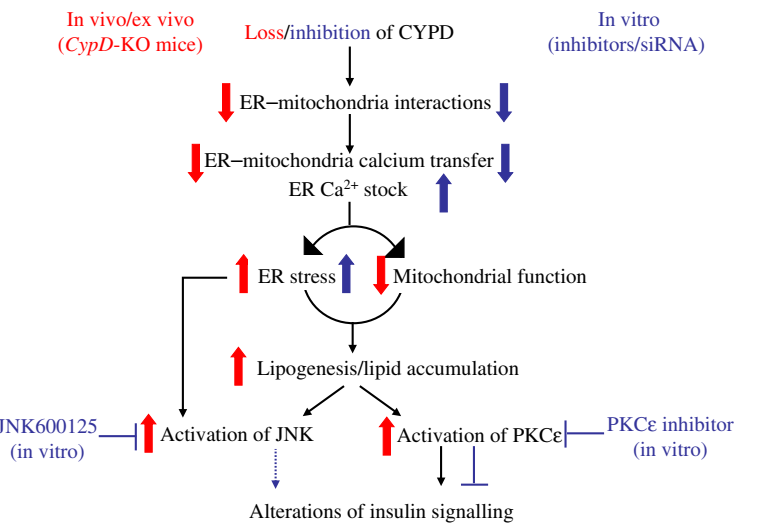

Fig. 8 Involvement of $\mathrm{PKC} \varepsilon$, but not of JNK, in $C Y P D$ siRNA-mediated alteration of insulin signalling in $\mathrm{HuH7}$ cells. (a, b) Representative western blot and quantitative analysis of basal (white bars) and insulin-stimulated (black bars) PKB phosphorylation in $\mathrm{HuH7}$ cells silenced for $C Y P D$, in the presence or absence of an inhibitor of JNK (SP600125, $10 \mu \mathrm{mol} / \mathrm{l}$ ) (a) or PKCE (specific peptide inhibitor of PKCE, $10 \mu \mathrm{mol} / \mathrm{l}$ ) (b). For western blots shown in (b), four parts of the same gel are shown. ${ }^{*} p<0.05$ and ${ }^{* *} p<0.01$ vs basal; ${ }^{\dagger} p<0.05$ vs respective Co siRNA $(n=3)$. (c) Schematic representation of the effects of loss/inhibition of CYPD on hepatic insulin resistance. Red, in vivo and ex vivo observations; blue, in vitro observations in $\mathrm{HuH7}$ cells

insulin action and demonstrated that disruption of MAM integrity in the liver of $C y p D-\mathrm{KO}$ mice can induce hepatic insulin resistance [10]. In the present study, we further investigated the underlying mechanisms and showed that the disruption of $\mathrm{Ca}^{2+}$ transfer from ER to mitochondria in the liver of $C y p D-\mathrm{KO}$ mice is likely the molecular consequence of MAM disruption, accounting for defective insulin action in the liver.

ER-mitochondria contact points are known to be enriched in $\mathrm{Ca}^{2+}$-handling proteins and chaperones and to generate microdomains with a high $\mathrm{Ca}^{2+}$ concentration [3]. We provide in vivo and in vitro evidence that pharmacological or genetic inhibition of the mitochondrial chaperone CYPD results in modifications of ER-mitochondria $\mathrm{Ca}^{2+}$ transfer: (1) via CYPD binding to the mitochondrial inner membrane since its actions are inhibited by CsA and NIM811 and (2) via an interaction with the VDAC1GRP75-IP3R1 $\mathrm{Ca}^{2+}$-channelling complex. These data are consistent with our recent study demonstrating that inhibition of CYPD in cardiomyocytes decreased the $\mathrm{Ca}^{2+}$ transfer from ER to mitochondria through IP3R under normoxic conditions [9]. Furthermore, CYPD-mediated disruption of ER-mitochondria cross-talk seems related rather to a dissociation of organelle than to a modification of protein expression at MAM interface, and seems independent of a modification of both $M c u$ and Serca $2 b$ mRNA levels. This new physiological role of CYPD in ER-mitochondria communication should now be considered as clinically relevant because inhibitors of CYPD function are either already used in clinical practice (e.g. CsA in transplanted patients) or are being developed (e.g. for treatment of hepatitis $\mathrm{C}$ virus or HIV infections) [24].

Our findings also point to a fundamental mechanism by which reduced structural and functional ER-mitochondria interactions alter organelle function and subsequently inhibit insulin's action, leading to hepatic insulin resistance. We thus propose a model by which the loss of CYPD can induce liver insulin resistance (Fig. 8c). Invalidation of CYPD alters ERmitochondria interactions and $\mathrm{Ca}^{2+}$ exchange, leading to increased ER $\mathrm{Ca}^{2+}$ storage and ER stress. It is likely that the loss of CYPD-mediated uncoupling of the ER from the mitochondria is compensated by increased levels of the ER proteinfolding machinery and $\mathrm{Ca}^{2+}$ to re-establish ER homeostasis, as previously reported $[4,5,21]$. At the same time, lack of $\mathrm{Ca}^{2+}$ transfer to mitochondria in $C y p D-\mathrm{KO}$ mouse hepatocytes can reduce mitochondrial respiration, as also seen in a recent study demonstrating that constitutive low-level IP3R-mediated $\mathrm{Ca}^{2+}$ delivery to mitochondria is essential to maintain normal cellular bioenergetics [22]. The chronic disturbance of $\mathrm{Ca}^{2+}$ homeostasis likely maintains the activation of unfolded protein response in liver of CypD-KO mice, despite increased GRP78 expression. Subsequently, both ER stress and mitochondrial dysfunction may contribute to an increase in hepatic lipid levels. Indeed, fat accumulation in the liver of $C y p D-\mathrm{KO}$ mice seems to be related to both a reduction in lipid oxidation (based on reduction in Cpt1 expression) and an induction of de novo lipogenesis (based on induction of lipogenic genes), whereas esterification of lipid (based on the absence of change in circulating NEFA levels) and export of lipids (based on induction of $A p o B$ and Mttp genes) from liver should not be altered. The induction of de novo lipogenesis is in agreement with the activation of both PERK and IRE1 branches of the unfolded protein response in the liver of $C y p D-\mathrm{KO}$ mice, as both pathways were shown to activate the lipogenic transcription factor SREBP-1c [25-27]. Particularly, accumulation of DAG likely contributes to $\mathrm{PKC} \varepsilon$ activation in the liver of $C y p D-\mathrm{KO}$ mice. Together, these metabolic stresses would consequently result in alterations of hepatic insulin signalling and in the deterioration of glucose homeostasis in CypD-KO mice. Indeed, both induction of ER stress and accumulation of intracellular lipids have been involved in hepatic insulin resistance. Activation of JNK has been shown in ER stress-mediated hepatic insulin 
resistance [20] and activation of $\mathrm{PKC} \varepsilon$ has been related to DAG-induced hepatic insulin resistance [23]. In CypD-KO mice, hepatic insulin resistance appears to be mainly secondary to ER stress modulation of hepatic lipogenesis and subsequent DAG-mediated activation of $\mathrm{PKC} \varepsilon$ rather than to ER stressinduced JNK activation, at least in vitro. Whereas an unspecific effect of inhibitors could not be excluded, this result fits well with the mechanisms recently proposed for another mouse model of insulin resistance [28], as well as with recent data in human liver [23]. Nevertheless, we cannot exclude the possibility that other players could be involved in the insulinresistant phenotype of $C y p D-K O$ mice. Particularly, as absolute cytosolic $\mathrm{Ca}^{2+}$ levels were increased in $C y p D$-KO mouse hepatocytes, we cannot exclude the participation of $\mathrm{Ca}^{2+}$-sensitive kinases and/or phosphatases in the alteration of insulin signalling, as suggested by others studies [29, 30]. As disruption of MAM integrity has been observed in liver of different mouse models of obesity and type 2 diabetes [10], it is likely that the unexpected role of inter-organelle $\mathrm{Ca}^{2+}$ exchange in triggering hepatic insulin resistance might be extrapolated to these models. In agreement, the direct inhibition of IP3R, independently of CYPD, also induced ER stress and altered hepatic insulin signalling, suggesting that the proposed mechanism is a more general model and not restricted to the $C y p D-\mathrm{KO}$ mouse model. Nevertheless, as controversy exists in this topic [31], further studies are required to clarify the role of inter-organelle $\mathrm{Ca}^{2+}$ exchange in triggering hepatic insulin resistance in obesity.

In conclusion, our data demonstrate that CYPD is an important regulator of MAM integrity and subsequently of $\mathrm{Ca}^{2+}$ exchange at the MAM interface, and provide the first evidence that IP3R-mediated $\mathrm{Ca}^{2+}$ transfer from ER to mitochondria is an essential cellular process involved in the control of hepatic insulin action. Therefore, modulation of ER-mitochondria $\mathrm{Ca}^{2+}$ exchange may provide an exciting new avenue for treating hepatic insulin resistance.

Acknowledgements The authors would like to thank the Lyon-Est imaging center (CIQLE) and SFR Santé Lyon Est (CNRS UMS3453 - INSERM US7, Université Lyon 1, France) for access to technological platforms and Physiogenex (Labege, France) for providing hyperinsulinaemiceuglycaemic clamps.

Funding This work was supported by Servier Laboratories, Inserm, the ANR (ANR-07-PHYSIO-020-01 and ANR-11-BSV1-033-02 from MO and ANR-09-JCJC-0116 from JR) and the FRM (MO, AL).

Duality of interest The authors declare that there is no duality of interested associated with this manuscript.

Contribution statement JR and MO conceived and designed the study. JF, MP, EBe, ET, MAC, AD, AB, GT, BB, MM, PT, GV, MD, $\mathrm{EB}$ and LG contributed to the collection of data. JR, JF and MP analysed and interpreted the data. JR and MO wrote the paper. FZ, HV and AL contributed to study conception and reviewed the manuscript for important intellectual concept. All authors contributed to critical revisions and have read and approved the final version to be published. JR is responsible for the integrity of the work as a whole.

\section{References}

1. Giorgi C, De Stefani D, Bononi A, Rizzuto R, Pinton P (2009) Structural and functional link between the mitochondrial network and the endoplasmic reticulum. Int J Biochem Cell Biol 41:18171827

2. Szabadkai G, Bianchi K, Varnai P et al (2006) Chaperone-mediated coupling of endoplasmic reticulum and mitochondrial $\mathrm{Ca}^{2+}$ channels. J Cell Biol 175:901-911

3. Rizzuto R, Pinton P, Carrington W et al (1998) Close contacts with the endoplasmic reticulum as determinants of mitochondrial $\mathrm{Ca}^{2+}$ responses. Science 280:1763-1766

4. de Brito OM, Scorrano L (2008) Mitofusin 2 tethers endoplasmic reticulum to mitochondria. Nature 456:605-610

5. Simmen T, Aslan JE, Blagoveshchenskaya AD et al (2005) PACS-2 controls endoplasmic reticulum-mitochondria communication and Bid-mediated apoptosis. EMBO J 24:717-729

6. Csordas G, Renken C, Varnai P et al (2006) Structural and functional features and significance of the physical linkage between ER and mitochondria. J Cell Biol 174:915-921

7. van Vliet AR, Verfaillie T, Agostinis P (2014) New functions of mitochondria associated membranes in cellular signaling. Biochim Biophys Acta 1843:2253-2262

8. Woodfield K, Ruck A, Brdiczka D, Halestrap AP (1998) Direct demonstration of a specific interaction between cyclophilin-D and the adenine nucleotide translocase confirms their role in the mitochondrial permeability transition. Biochem J 336:287-290

9. Paillard M, Tubbs E, Thiebaut PA et al (2013) Depressing mitochondria-reticulum interactions protects cardiomyocytes from lethal hypoxia-reoxygenation injury. Circulation 128:1555-1565

10. Tubbs E, Theurey P, Vial G et al (2014) Mitochondria-associated endoplasmic reticulum membrane (MAM) integrity is required for insulin signaling and is implicated in hepatic insulin resistance. Diabetes 63:3279-3294

11. Betz C, Stracka D, Prescianotto-Baschong C, Frieden M, Demaurex N, Hall MN (2013) Feature article: mTOR complex 2-Akt signaling at mitochondria-associated endoplasmic reticulum membranes (MAM) regulates mitochondrial physiology. Proc Natl Acad Sci U S A 110:12526-12534

12. Sebastian D, Hernandez-Alvarez MI, Segales J et al (2012) Mitofusin 2 (Mfn2) links mitochondrial and endoplasmic reticulum function with insulin signaling and is essential for normal glucose homeostasis. Proc Natl Acad Sci U S A 109:5523-5528

13. Hagiwara A, Cornu M, Cybulski N et al (2012) Hepatic mTORC2 activates glycolysis and lipogenesis through Akt, glucokinase, and SREBP1c. Cell Metab 15:725-738

14. Ye R, Ni M, Wang M et al (2011) Inositol 1,4,5-trisphosphate receptor 1 mutation perturbs glucose homeostasis and enhances susceptibility to diet-induced diabetes. J Endocrinol 210:209-217

15. Schinzel AC, Takeuchi O, Huang Z et al (2005) Cyclophilin D is a component of mitochondrial permeability transition and mediates neuronal cell death after focal cerebral ischemia. Proc Natl Acad Sci U S A 102:12005-12010

16. Takahashi A, Camacho P, Lechleiter JD, Herman B (1999) Measurement of intracellular calcium. Physiol Rev 79:1089-1125

17. Berry MN, Friend DS (1969) High-yield preparation of isolated rat liver parenchymal cells: a biochemical and fine structural study. J Cell Biol 43:506-520 
18. Nicolli A, Basso E, Petronilli V, Wenger RM, Bernardi P (1996) Interactions of cyclophilin with the mitochondrial inner membrane and regulation of the permeability transition pore, and cyclosporin A-sensitive channel. J Biol Chem 271:2185-2192

19. Zhao Q, Wang J, Levichkin IV, Stasinopoulos S, Ryan MT, Hoogenraad NJ (2002) A mitochondrial specific stress response in mammalian cells. EMBO J 21:4411-4419

20. Ozcan U, Cao Q, Yilmaz E et al (2004) Endoplasmic reticulum stress links obesity, insulin action, and type 2 diabetes. Science 306:457-461

21. Bravo R, Vicencio JM, Parra V et al (2011) Increased ERmitochondrial coupling promotes mitochondrial respiration and bioenergetics during early phases of ER stress. J Cell Sci 124:2143-2152

22. Cardenas C, Miller RA, Smith I et al (2010) Essential regulation of cell bioenergetics by constitutive $\mathrm{InsP} 3$ receptor $\mathrm{Ca} 2+$ transfer to mitochondria. Cell 142:270-283

23. Kumashiro N, Erion DM, Zhang D et al (2011) Cellular mechanism of insulin resistance in nonalcoholic fatty liver disease. Proc Natl Acad Sci U S A 108:16381-16385

24. Quarato G, D'Aprile A, Gavillet B et al (2012) The cyclophilin inhibitor alisporivir prevents hepatitis $\mathrm{C}$ virus-mediated mitochondrial dysfunction. Hepatology 55:1333-1343

25. Li H, Min Q, Ouyang C et al (2014) AMPK activation prevents excess nutrient-induced hepatic lipid accumulation by inhibiting
mTORC1 signaling and endoplasmic reticulum stress response. Biochim Biophys Acta 1842:1844-1854

26. Ning J, Hong T, Ward A et al (2011) Constitutive role for IRE1aXBP1 signaling pathway in the insulin-mediated hepatic lipogenic program. Endocrinology 152:2247-2255

27. Zhang C, Chen X, Zhu RM et al (2012) Endoplasmic reticulum stress is involved in hepatic SREBP-1c activation and lipid accumulation in fructose-fed mice. Toxicol Lett 212:229-240

28. Jurczak MJ, Lee AH, Jornayvaz FR et al (2012) Dissociation of inositol-requiring enzyme (IRE1alpha)-mediated c-Jun N-terminal kinase activation from hepatic insulin resistance in conditional $\mathrm{X}$ box-binding protein-1 (XBP1) knock-out mice. J Biol Chem 287: 2558-2567

29. Ozcan L, Wong CC, Li G et al (2012) Calcium signaling through CaMKII regulates hepatic glucose production in fasting and obesity. Cell Metab 15:739-751

30. Wang Y, Li G, Goode J et al (2012) Inositol-1,4,5-trisphosphate receptor regulates hepatic gluconeogenesis in fasting and diabetes. Nature 485:128-132

31. Arruda AP, Pers BM, Parlakgül G et al (2014) Chronic enrichment of hepatic endoplasmic reticulum-mitochondria contact leads to mitochondrial dysfunction in obesity. Nat Med 20:1427-1435 